\title{
Experience of Care as a Critical Component of Health System Performance Measurement: Recommendations for Moving Forward
}

\author{
To \\ INVITED ESSAY \\ Kerry Kuluski, MSW, $\mathrm{PHD}$ \\ Scientist, Bridgepoint Collaboratory of the Lunenfeld-Tanenbaum Research Institute \\ Sinai Health System \\ Assistant Professor, Institute of Health Policy, Management and Evaluation \\ Dalla Lana School of Public Health, University of Toronto \\ Toronto, $\mathrm{ON}$ \\ Michelle L.A. Nelson, $\mathrm{PHD}$ \\ Scientist, Bridgepoint Collaboratory of the Lunenfeld-Tanenbaum Research Institute \\ Sinai Health System \\ Assistant Professor, Institute of Health Policy, Management and Evaluation \\ Dalla Lana School of Public Health, University of Toronto \\ Toronto, $\mathrm{ON}$ \\ C. Shawn Tracy, $\mathrm{PhD}(\mathrm{C})$ \\ Research Associate, Sinai Health System \\ Toronto, ON \\ Carole Ann Alloway \\ Co-Founder, Family Caregivers Voice \\ Toronto, ON
}


Experience of Care as a Critical Component of Health System Performance Measurement

\title{
Charles Shorrock
}

Customer Experience Leader and Patient Experience Advisor

Ontario Patient Ombudsman's Office

Toronto, ON

Sara Shearkhani, MA, $\mathrm{PHD}(\mathrm{C})$

Institute of Health Policy, Management and Evaluation

Dalla Lana School of Public Health, University of Toronto

Co-Founder, Family Caregivers Voice

Toronto, $\mathrm{ON}$

Ross E. G. Upshur, MA, MD, MSc, CCFP, FRCPC

Head, Division of Clinical Public Health

Dalla Lana School of Public Health, University of Toronto

Scientific Director, Bridgepoint Collaboratory of the Lunenfeld-Tanenbaum Research Institute Sinai Health System

Professor, Department of Family and Community Medicine, University of Toronto

Toronto, ON

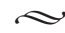

\begin{abstract}
People's experiences can provide critical guidance on how to better meet their quality of life and care needs and deploy resources more appropriately. To maximize the utility of experience data and to advance the current debate, we present four recommendations: (1) measuring experiences outside the healthcare system can provide insight into what needs to change within the healthcare system; (2) focusing on patient experience is necessary but insufficient, (family) caregiver insights and experiences require attention and can provide insight into the needs of the patient; (3) moving from "one time/single sector" measurement of experience to iterative, ongoing measurement across sectors better reflects the true lived experience of patients (especially those with complex care needs) and their caregivers; and (4) embedding measurement within engagement-capable environments that adequately resource patients, caregivers, and providers to work together is required to move from collection to meaningful change. Applying these recommendations requires a longer-term vision, shifting from provider-centred to person-centred models of care, and a deep understanding of the structural, cultural, and normative barriers to measuring care experiences.
\end{abstract}

\section{Background and Introduction}

The Institute for Health Improvement's "triple aim" asserts that high performing health systems require the simultaneous achievement of better health outcomes, lower costs and better experience of care (Berwick et al. 2008). The importance of achieving such aims is fueled by population aging, growing 
complexity in consumer care needs, concerns over healthcare system costs and sustainability and a growing recognition that healthcare systems (oriented to deliver acute, episodic and single disease-oriented care) are out of step with the needs of patients and their caregivers (Gill et al. 2016; Marengoni et al. 2011; Paddison et al. 2015; Salsbury 2012; Tinetti et al. 2012).

The better care experience aim has been gaining attention in policy and research circles with studies noting that experiences tend to worsen as care needs become more complex (Vogeli et al. 2007). As care needs increase, so too does the burden on family caregivers (which often includes friends and neighbours), who make enormous personal sacrifices in their roles as unpaid providers of care. In addition, clinicians have few tools and guidelines to support the management of complex care (Nelson et al. 2016; Osborn et al. 2015). To that end, optimizing health systems is contingent on not only creating opportunities for patients and their caregivers to comfortably share their experiences, but simultaneously increasing the capacity of providers and organizations to enable a culture of patient and family engagement (Baker et al. 2016).

Capturing the care experience as a critical component of health system performance measurement has gained traction over the last few decades (Berwick et al. 2008). There is growing consensus that measuring the care experience is important to meet the care needs of patients and families (Salisbury 2012), but it is unclear if this is done consistently and appropriately. While healthcare systems have moved towards embracing principles of "patient and person centredness," they have neglected, at the same time, to recognize and address the measurement challenges.

In this lead paper, we bring together the combined expertise of research, clinical care, and lived experience (patient and caregiver perspectives) to propose patient and caregiver experience as a critical component of performance measurement. We reflect on what elements of the experience should be considered; whose experience of care should be captured and why; where and when experiences should be captured; as well as how to move this agenda forward within the context of healthcare systems' constraints. If, as we argue, healthcare system improvement requires increased attention on bettering the experience of care, we hope to initiate a discussion of how best to proceed with measuring care experience in a way that leads to meaningful change for people and their caregivers.

\section{As care needs increase, so too does the burden on family caregivers ... in their roles as unpaid providers of care.}

\section{"What Elements of the Care Experience should we Measure?"}

Key message: Measuring experiences outside the formal healthcare system can provide insight on what needs to change within the healthcare system

There is a wide range of health system performance measures, and we share a few examples here. Performance measures may capture population health (e.g., mortality), clinical outcomes (e.g., pre- and posttreatment symptoms), clinical processes (e.g., appropriate prescribing), organizational targets (e.g., length of hospital stay), people's experiences - typically patients (e.g., access to care, quality of relationships with care providers), and measures of efficiency and productivity (e.g., value for dollars spent) (Smith et al. 2008). Moreover, there is growing adoption of, and attention to, measures that are patient reported (Jenkinson and Fitzpatrick 2013). 
We focus our critique on care experiences, a complex concept to measure given its lack of conceptual clarity (LaVela and Gallan 2014). Definitions of care experience, typically anchored to the patient, abound in the literature. To provide one example, the US Agency for Healthcare Research and Quality (AHRQ) states that patient experience "encompasses the range of interactions that patients have with the healthcare system, including their care from health plans, and from doctors, nurses, and staff in hospitals, physician practices, and other healthcare facilities. The terms patient satisfaction and patient experience are often used interchangeably, but they are not the same thing" (AHRQ 2016). This definition, like many others, explicitly focuses on experiences within the formal healthcare system (i.e., largely facility-based care including hospitals). This narrow conceptualization of the patient experience excludes a vast array of other experiences that impact health that occur outside the healthcare system. It is now widely accepted that health status and health outcomes are determined by factors both inside and outside the formal healthcare system. Measuring experiences outside the healthcare system that impact health necessarily takes us into the realm of social determinants (e.g., housing, income, etc.) and tipping points (the things that happen before people land in the healthcare system), which seem important to capture. If our ultimate goal is to improve the health of our populations, reduce per capita costs, and enhance people's experience simultaneously, we need to measure beyond the healthcare system experience.

AHRQ's patient experience definition highlights a key point - that satisfaction does not equal experience. Satisfaction measures may capture level of contentment with a particular service or provider, or the extent to which expectations were met. The challenge with this approach is that people may have low expectations to begin with, given their recognition of the constraints of the system and its limited capacity. Also, satisfaction does not provide insight into what specifically needs to change and how; whereas, experience aims to capture what happened and how it made the person feel, providing greater guidance for quality improvement. This is illustrated in work conducted by Glenn Robert (2013) which demonstrates that a person may indicate high satisfaction with their care through a quantitative "tick the box" data collection method, but when given the opportunity to elaborate, through a narrative or open-ended response, a very different picture may emerge. This contradiction highlights the importance of the patient narrative, as opposed to just quantitative measures of performance measurement. Indeed, mixed methods approaches to capturing experience are highly recommended as a best practice for data collection (Lees 2011).

A recent qualitative study of patients with complex care needs and their caregivers conducted by Lafortune et al. (Lafortune et al. 2017) noted that common assessment tools used in healthcare tend to exclude things that are important to people including: informal caregiver and family considerations (degree of inclusion in healthcare decisions, location to patient and capacity to provide care given other commitments), insider healthcare knowledge (enhanced when patients or caregivers have current or former roles in the health system), and patients' attitudes based on previous healthcare experiences (negative experiences can lead to lack of future engagement). Capturing these domains requires open ended or narrative approaches and can provide important context to understanding people's experiences. While taking such an approach to data collection in healthcare may be timely and costly, there may be opportunities 
to capture fuller narratives from a subset of patients and caregivers, add open-ended questions to existing tools, or to reorient data collection approaches focusing on those with the greatest return (value).

In addition to Lafortune's findings, there are other measures and domains that may be worthy of consideration for broad scale health system adoption. Already present in the Canadian Institute for Health Information's (CIHI) Canadian Patient Experiences Survey - Inpatient (for acute care hospitals) is the relational aspects of care - including whether or not patients feel that they are heard by their providers, treated with respect, and explained things in a way they can understand (CIHI 2015). These questions should also be directed to caregivers. Further, capturing patient and caregiver priorities, or goals should be considered particularly as care needs, morbidities and symptoms multiply (Kuluski et al 2013). The management of multimorbidity is complex, uncertain, and requires a number of trade-offs. Discussing and measuring goals of care, and goal alignment among the care team, patients and families can importantly help to prioritize complex care regimens as well as life priorities and ensure that care is at least somewhat aligned to what people need (but also clinically safe and appropriate). A growing body of evidence suggests that when care is aligned with the needs and goals of patients and their families, better treatment adherence, improved self-management, appropriate use of resources, and better care experiences result (Belle Brown et al. 2003; Doyle et al. 2013). An important addition is assessment of whether goals were met.

$$
\begin{aligned}
& \text { Decades of research point to } \\
& \text { the significant contributions } \\
& \text { of caregivers... }
\end{aligned}
$$

\section{"Whose Experiences are We Measuring and Why?"}

Key message: Measuring patient experience is necessary but insufficient; the experience of caregivers should also be captured.

There is widespread consensus that measuring the patient experience is integral to making improvements in care and several countries are now pursuing this activity (Coulter et al. 2014). We propose that attention should also be given to the experience of caregivers - that is, the unpaid family, friends, and neighbours who are, more often than not, closely connected to the patient.

Decades of research point to the significant contributions of caregivers, including the hours of care provided, the range of supports given, from instrumental activities (transportation, preparing meals, and managing finances) to heavier personal care (bathing, toileting, and medical treatments such as oxygen therapy and wound care). Through their contributions, caregivers save the health system millions of dollars each year (Hollander et al. 2009), but unfortunately, and too commonly, do so at the expense of their own health and quality of life (Perkins et al. 2013; Sinha 2013; Turcotte 2013). The wealth of knowledge they hold on the needs, preferences, and conditions of patients becomes integral when interacting with the healthcare system; however, they are seldom considered to be part of the care team.

More often than not, patients and caregivers are the only source of reliable knowledge amid the various provider interactions, treatment alterations, medication changes, care transfers, and other care episodes that occur. Caregivers typically hold a great deal of "tacit" or "insider" knowledge of the patient including their unique behaviors, habits, triggers, preferences, and patterns that can lead to a fulsome understanding of a person - a level of knowledge necessary to improve their care and experience. 
Reinders (2010) asserts that "tacit" knowledge is grounded in strong interpersonal relationships, is hard to measure and less likely to emerge between formal care providers and patients. Formal care providers are embedded within environments that incentivize short visits, limited follow-up, poor provider continuity, and distinctive epistemic and normative cultures which may not coincide with collaborative approaches to care. For this reason, we argue that caregivers are more likely (than formal providers), by virtue of their continuous relationship and role, to hold this type of knowledge of the patient. While there are times when incorporating the caregiver perspective of the patient may be deemed inappropriate, difficult, or even contentious (because of patient preferences, disagreements between multiple caregivers, in cases of strained and abusive relationships, among other factors), including their perspective provides an important opportunity for healthcare providers to better understand the patient (Kurtz et al. 1996; Roydhouse and Wilson 2017) and potentially improve their experience.

It is also important to learn more about the caregiver. Caregiving is associated with many positive outcomes (Greenwood et al. 2009), but the myriad negative impacts including poor physical and mental health outcomes, opportunity costs (Turcotte 2013) and high degree of unmet need - suggest that assessing their experience in and of itself is important. Despite little monitoring of caregiver experiences in health systems (de Silva 2013), there are some promising developments. A recent Canadian knowledge synthesis of patient reported outcome and experience measures outlined a number of measures that have been used in acute care settings, including those that are applicable to caregivers. The challenge is that there is little understanding of how these measures inform health services improvements and clinical decision-making (Sawatzky et al. 2015). A promising development in the UK, stemming from the Carers Act, entitles all adult caregivers of ill or disabled persons to a carers assessment (Carers UK 2014), regardless of whether or not the patient has been assessed. The carer assessment examines the physical and emotional needs of caregivers, their capacity to provide care, as well as their service needs. This unique policy shift provides a basis to better understand a caregiver's experience and needs and can serve as a model for other jurisdictions seeking a similar path.

Caregiver experiences are uniquely shaped by the relationships they have with the care recipient, their stage of life, stage of caregiving, social roles and responsibilities, culture, economic stability, living arrangements, among other factors. These factors shape capacity to provide care, ability to access knowledge and resources, and a willingness to both seek and provide support. Capturing their experience provides an opportunity to acknowledge their key contribution as partners in the health system and may protect their health and role. In doing so, we move closer to a patient- and caregiver-oriented health system. As noted by one of our caregiver co-authors, supporting the caregiver supports the patient to achieve their best health outcome.

\section{"When and Where do We Measure Experience?"}

Key message: Moving from "one time/single sector" measurement of experience to iterative, ongoing measurement across sectors would better reflect the true lived experience of care, especially for patients with complex care needs and their caregivers.

Work to date regarding when to measure the experience of care suggests a dichotomy: either during the care experience or after 
the care experience (LaVela 2014; Bjertnaes 2013), with corresponding processes and purposes. While there are strengths and limitations to both approaches, greater strategic thinking is required regarding the format and timing of measurement to ensure it is purposeful, designed to capture data of interest, and ideally matches the patient and caregivers' experiences with the healthcare system. Different measurement processes and time frames will provide different types of experience data that can be used for different purposes.

"Real time/in situ" data collection can help providers gain insight into the experience of care for individual patients and caregivers with the ability to determine which needs and goals are being met and which are being left unmet. Measuring experience in real time could allow for adaptations to the experience during the experience to better meet patient and caregiver needs. Over and above the improvement in the experience of care, this approach has the added potential benefit of also improving outcomes and enhancing value.

The value of "post-experience" assessment lies in the collection of data that can be quantified as a result of a larger sample size, which can then be used for performance benchmarking and compared to patient outcomes. The challenge, however, is to implement this measurement in a timely fashion. Work to date suggests that patients and caregivers struggle to delineate which experience specifically they are evaluating when asked to respond after a lengthy delay (Manary et al. 2013), and may be subject to sources of bias (recall, family discussion, etc.) (LaVela 2014). Bjertnaes et al. (2012) note that greater passage of time since the encounter is correlated with a poorer experience being reported. Although post-experience measurement can be used to improve clinical services and systems, an important limitation of post-experience measurement is that the opportunity to use the feedback to improve the experience for that specific patient has been missed.

Combining the strengths of "real time" actionable data with post-experience data can contribute different insights into elements of the experience. Real-time evaluation is implemented to provide information regarding necessary revisions or modifications to the program or process addressing the question "how are we doing?" (Patton et al. 2015). These types of activities are often used to foster continuous improvement within an ongoing activity. Post-experience evaluations generally occur at the completion of a cycle, assessing the outcome of the process by asking the question "how did we do?"

As care needs evolve over time, the setting of care can change; as such, the "when" and the "where" of measuring the experience of care are intricately connected. Regardless of timing and purpose, collecting experience data for a single isolated health event can be relatively straightforward. The patient and caregiver can more easily recall the experience and report their perspectives on the experience. Where the measurement of experience becomes complex is in patient populations who are continuously accessing healthcare services across multiple sectors and settings. The issue of care transitions is among the thorniest of challenges to system planners and policy makers. But if the prevailing approach to measuring care experience has been largely within organization and within sector, then perhaps it is not surprising that transitions across organizations and across sectors have emerged as such a challenge. In Canada, the need has been identified for a set of standard measures specific to the experience of care when care involves multiple transitions (HQO 2016). This work, however, must be accelerated given the growing numbers of people 
with multiple complex health problems and the myriad transitions and episodes of care experienced cross-sectorally. Without crosssectoral data collection, key areas of experience and opportunities for improvement will be lost, particularly around transitions in care.

\section{... there is evidence to suggest that varied perceptions exist as to the time frame encompassed within "the experience of care"...}

The approach to system performance measurement that currently predominates (i.e., measuring discrete episodes of care, usually after the fact) mirrors the structure of the healthcare system itself (i.e., fragmented, single-disease focus, episodic care). For the highest cost user - perhaps they are perpetually in situ but are inundated with post-evaluation requests - and we miss the opportunity to understand/address/capture the experience of being a patient within and across multiple sectors, providers and settings. Interestingly, there is evidence to suggest that varied perceptions exist as to the time frame encompassed within "the experience of care" for a given episode of care. For example, a recent Australian study found that hospital care providers define the care experience as starting at admission and ending at discharge, whereas for care recipients the hospital care experience included an extended period both before admission and after discharge (Edwards et al. 2014).

Sector-specific surveys are the most common method of collecting patient experience in health systems. England's NHS has been a leader in this field, starting in the 1980s when the NHS Management Inquiry recommended the use of patient experience surveys to determine how well services were working (Graham and Woods 2013). In 2001, there was a countrywide cross-sectoral mandate to conduct patient care experience surveys (de Silva 2013). While other countries have followed suit, few, including Canada, have compulsory, systematic collection of patient experience across the health system. It is important, however, to note the progress that has been made. Focusing on Canada, CIHI has developed a standardized hospital in-patient experience survey that is available to all acute care hospitals across Canada, but its adoption is not mandatory (CIHI 2015). An accompanying procedure manual and toolkit to guide data collection and reporting has been developed for ease of implementation and to support comparability between participating hospitals. The questions were adapted from the Consumer Assessment of Healthcare Providers and Systems Surveys (a suite of patient experience surveys developed in the US) and further adapted following input from an inter-jurisdictional committee of experts.

While the questions on the CIHI survey capture important domains of experience (including the relational aspects of care referred to earlier), we see some important gaps. For example, there is little attention to factors outside of hospital. Indeed, it is a hospital experience survey, but certain factors, if not examined, could place patients at risk of hospital readmission. These include caregiver willingness, readiness, and capacity; access and transfer to home care; and social supports post-discharge. Patient experience surveys for primary care and long-term care sectors are currently in development, but may miss experience of "the system" and transitions through sectors as they will no doubt focus on sector specific experience. Health Quality Ontario's patient experience measurement strategy recognizes this limitation and has prioritized transitions and cross-sectoral experience 
of care as a core area for future development (HQO 2016).

Measurement of experience over time and across settings is essential in providing insight into improvement strategies - but requires cross-sector collaborations, sharing in the collection and use of the data. In 2010, The Change Foundation (2010) concluded, "Provider-focused surveys by their nature cannot look across to other providers or sectors. Given the sampling methodologies and protocols, providers would not in most cases be able to identify which of their patients received care from other providers for a related condition, the nature of the related care, and when it might have happened" (p. 12). More recently, HQO notes that data-sharing mechanisms to enable use of patient experience data across organizations and sectors are essential (2017). Our quality improvement efforts hinge on the quality of our data. Therefore, on a systems level, it is critical to capture data over time and across all settings of care. But this is dependent upon the development of accountability processes and incentives for health system performance that embrace principles of patientand family-centred care and cross-sectoral thinking and working.

$$
\begin{aligned}
& \text {... the inherent implication is that } \\
& \text { the data collected will be used to } \\
& \text { improve the patient and caregiver } \\
& \text { experience, and... the system. }
\end{aligned}
$$

\section{"How do We Move Forward?"}

Key message: Embedding measurement within engagement-capable environments that adequately resource patients, caregivers, and providers to work together is required to move from data collection to meaningful change.
When we measure patient and caregiver experience, we set an expectation that something will be done in response to this data collection. What is our commitment as a system, organization, or provider? Are we adequately prepared to not only begin the conversation, but actively participate and see it through? Data collection implies a purpose. In the case of performance measurement, the inherent implication is that the data collected will be used to improve the patient and caregiver experience, and through that, the overall performance of the system.

To avoid gathering information with no valuable output (Coulter et al. 2014), we need engagement-capable environments, a concept described by Baker et al (2016). The development of engagement-capable environments needs more than external stimuli - policy directives, performance targets, and funding incentives. To be engagement capable, a number of internal factors need attention, including the creation and articulation of a strong organizational vision, the building up of leadership capacity at various levels of the organization, attention to the satisfaction of care providers and the resources they need to work more effectively with patients and families, dedicated resources to support patient and family involvement (from clinical encounters to organizational committee and leadership opportunities), and ongoing performance reporting to measure progress (Coulter et al. 2014; Luxford et al. 2011). The challenge is that healthcare systems are constantly seeking timely and implementable solutions, leaving little time to determine what is truly needed from system users. Within constrained environments, there is little time to develop the infrastructure required to enable and support a culture shift. Indeed, the significant time it takes to shift from provider-centred organizations to person-centred organizations is one of the key cited barriers to implementation (Luxford et al. 2011). 
While many agree that capturing experience as a measure of system performance is important, critics assert that patients lack the necessary expertise to evaluate care quality. Critics also express that a focus on improving the patient experience encourages providers to respond to patient preferences leading to care that could be inappropriate and ineffective and that there is a trade-off between personcentred care, high quality clinical care (Anhang Price 2015) and other performance targets such as timely "throughputs" (hospital length of stay targets). Indeed, it is important that the pendulum does not swing too far in one direction; person-centred care and valuing the voice and perspectives of patients and caregivers does not mean doing everything that patients and caregivers want. Person-centred care must occur in a context that supports collective and sound decision-making among providers with patients and caregivers.

If we move toward the systematic (and systemic) collection of patient and family experience data, we should also take time to examine what we currently measure and whether or not it is useful (for patients, caregivers, clinicians, administrators, and policy planners) in an effort to minimize measurement fatigue and optimize the time and efforts of users and providers of care. Particularly, if we support a movement toward iterative and ongoing data collection, from people and families, across time and sectors (to understand a full continuum of care experience as opposed to specific, disjointed care episodes), attention to the time, effort, and cost is required.

In terms of metric development, it is important to question whether current measures are adequate to the task. True engagement would require patients, healthcare providers, caregivers, policy makers, and researchers to be involved in the critical rethinking of measures and their usefulness in capturing diverse experiences in increasingly heterogeneous populations. As the population becomes more complex, reliance on standardized measures becomes more problematic. Rather than pretending our current measures are doing the work they are intended to do, we may envision a "grand challenge" in the co-creation of meaningful measurement tools.

Another consideration is to discontinue sector-specific experience measures and follow the lead of the aforementioned carers assessment, which provides an overarching snapshot of experience that is relevant across time and place.

Perhaps the quickest win is to leverage large data platforms, available through data warehouses such as Ontario's Institute for Clinical Evaluative Sciences, by adding patient and caregiver experience data in an effort to systematically collect and analyze trends at a population level to inform change.

One example that highlights an opportunity to proceed with meaningful patient and caregiver experience data is Ontario's roll-out of hospital funding reform. Qualitybased procedures (QBPs; which refer to disease-specific services/best practices) have been implemented across Ontario's hospitals and refer to funding tied to specific disease groups. Whereas current performance measures focus on data elements available in administrative datasets, such as mortality and readmissions, there is an important opportunity to also capture patient and caregiver experiences and needs. For example, inviting a subset of patients and their caregivers in each QBP to participate in an interview during and following their episode of care would provide important context. Three key areas of experience that should be captured are: the extent to which their needs and priorities were identified and met during their hospital stay; their discharge 
concerns and whether or not these were captured and addressed; and community care needs (including both health and social care). Purposive sampling of patients who exceed intended length of stay targets or who are readmitted to hospital are two examples of patient subgroups that can be targeted to garner insight on the utility of the QBP in meeting the resource needs of patients and their caregivers.

Developing the infrastructure to support person-centred care and person-centred organizations may require significant up-front costs (e.g., changing care processes, training, new types of data collection, infrastructure to support information sharing, etc.). Perhaps the most challenging piece will be engaging with policy planners to identify and challenge the "hard-wired" processes that inhibit the development of engagement-capable environments, including reporting requirements and funding models that incentivize the collection of useless data or short care encounters, and privacy legislation that impedes efforts to share information across sectors, among others.

Despite these anticipated challenges, pursuing this journey can lead to significant long-term gains. Measuring patient and caregiver experiences will point to resources that people need as well as resources that are wasteful - used merely by default with little benefit to people or the system. Ultimately, experience data can be used to better direct current healthcare dollars to ensure greater value for dollars spent. While lower healthcare system costs is a possibility, similar costs with greater value for dollars spent is more likely. Importantly, we need to accept that, at least in the short term, pursuing a shift from providercentred care to person-centred care may "only" yield improvements in the quality of life for patients and their caregivers.

\section{Conclusion}

Improved experience of care is central to the IHI Triple Aim. Capturing experience is a necessary catalyst for meeting the quality of life and care needs of patients and caregivers and can provide the necessary information to deploy resources more appropriately to improve the value of dollars spent. To maximize the utility of experience data and to advance the current debate, we have presented four recommendations: (1) measuring experiences outside the healthcare system can provide insight into what needs to change within the healthcare system; (2) focusing on patient experience is necessary but insufficient, (family) caregiver insights and experiences require attention and can provide insight into the needs of the patient; (3) moving from "one time/single sector" measurement of experience to iterative, ongoing measurement across sectors better reflects the true lived experience of patients (especially those with complex care needs) and their caregivers; and (4) embedding measurement within engagement-capable environments that adequately resource patients, caregivers, and providers to work together is required to move from collection to meaningful change. Applying these recommendations requires a longerterm vision, shifting from provider-centred to person-centred models of care, and a deep understanding of the structural, cultural, and normative barriers to measuring care.

\section{References}

Agency for Healthcare Research and Quality (AHRQ). 2016. What is Patient Experience? Retrieved April 10, 2017. <https://www.ahrq. gov/cahps/about-cahps/patient-experience/index. html>.

Anhang Price, R., M.N. Elliott, P.D. Cleary, A.M. Zaslavsky and R.D. Hays. 2015. "Should Health Care Providers be Accountable for Patients' Care Experiences?" Journal of General Internal Medicine 30(2): 253-56. 
Experience of Care as a Critical Component of Health System Performance Measurement

Baker, G.R., M. Judd, C. Fancott and C. Maika. 2016. "Creating 'Engagement-Capable' Environments in Healthcare.” In Baker, G.R., M. Judd, C. Fancott and C. Maika, eds., Patient Engagement: Catalyzing Improvement and Innovation in Healthcare (pp. 11-34). Toronto, ON: Longwoods Publishing Corporation.

Belle Brown, J., M. Stewart, W. Westen and T. Freeman. 2003. "Introduction." In Stewart, M., J. Belle Brown, W.W. Weston, I.R. McWhinney, C.L. McWilliam and T.R. Freeman, Patient-Centered Medicine Transforming the Clinical Method (2nd ed., pp. 3-15). Abingdon, UK: Radcliffe Medical Press.

Berwick, D.M., T.W. Nolan and J. Whittington. 2008. "The Triple Aim: Care, Health, and Cost." Health Affairs 27(3): 759-69.

Bjertnaes, O.A. 2012. "The Association between Survey Timing and Patient-Reported Experiences with Hospitals: Results of a National Postal Survey." BMC Medical Research Methodology 12: 13.

Canadian Institute for Health Information (CIHI). 2015. Canadian Patient Experiences Survey - Inpatient Care Procedure Manual. Retrieved April 7, 2017. $<$ https://www.cihi.ca/sites/default/files/document/ cpes-ic-procedure-manual_2015_en.pdf>.

Carers UK. 2014. Carer's Assessment London, England. Retrieved April 18, 2017. <https://www. carersuk.org/help-and-advice/practical-support/ getting-care-and-support/carers-assessment>.

Coulter, A., L. Locock, S. Ziebland and J. Calabrese. 2014. "Collecting Data on Patient Experience Is Not Enough: They Must Be Used to Improve Care." BMJ 348: g2225.

de Silva, D. 2013. Measuring Patient Experience. Evidence Scan. Retrieved March 31, 2017. <http://www.health. org.uk/publication/measuring-patient-experience>.

Doyle, C., L. Lennox and D. Bell D. 2013. "A Systematic Review of Evidence on the Links between Patient Experience and Clinical Safety and Effectiveness." BMJ Open 3(1): pii: e001570.

Edwards, K., J. Duff and K. Walker. 2014. "What Really Matters? A Multi-View Perspective of One Patient's Hospital Experience." Contemporary Nurse 49: 122-36.

Gill, A., K. Kuluski, L. Jaakkimainen, G. Naganathan, R. Upshur and W.P. Wodchis. 2014. "Where Do We Go from Here?” Health System Frustrations Expressed by Patients with Multimorbidity, Their Caregivers and Family Physicians." Healthcare Policy 9(4): 73-89.

Graham, C. and P. Woods. 2013. "Patient Experience Surveys.” In: Ziebland, A.C., J.D. Calabrese and L. Locock, eds, Understanding and Using Health Experiences: Improving Patient Care (pp. 81-93). Oxford, UK: Oxford University Press.
Greenwood, N., A. Mackenzie, G.C. Cloud and N. Wilson. 2009. "Informal Primary Carers of Stroke Survivors Living at Home-Challenges, Satisfactions and Coping: A Systematic Review of Qualitative Studies." Disability Rehabilitation 31(5): 337-51.

Health Quality Ontario (HQO). 2016. Ontario Patient Experience Measurement Strategy. Retrieved April 7, 2017. <http://www.hqontario.ca/portals/0/ documents/system-performance/patient-experiencemeasurement-strategy-1608-en.pdf>.

Hollander, M.J., G. Liu and N.L. Chappell. 2009. "Who Cares and How Much? The Imputed Economic Contribution to the Canadian Healthcare System of Middle-Aged and Older Unpaid Caregivers Providing Care to the Elderly." Healthcare Quarterly 12(2): 42-49.

Jenkinson, C and R. Fitzpatrick. 2013. "Patient Reported Outcomes.” In Ziebland, S., A.C. Joseph, D. Calabrese, L. Locock, eds., Understanding and Using Health Experiences (pp. 72-80). Oxford, UK: Oxford University Press.

Kuluski, K., A. Gill, G. Naganathan, R. Jaakkimainen and W.P. Wodchis. 2013. "A Qualitative Descriptive Study on the Alignment of Care Goals between Older Persons with Multi-Morbidities, Their Family Physicians and Informal Caregivers." BMC Family Practice 14: 133.

Kurtz, M.E., J.C. Kurtz, C.C. Given and B. Given. 1996. "Concordance of Cancer Patient and Caregiver Symptom Reports.” Cancer Practice 4(4): 185-90.

Lafortune, C., J. Elliott, M.Y. Egan and P. Stolee. 2017. "The Rest of the Story: A Qualitative Study of Complementing Standardized Assessment Data with Informal Interviews with Older Patients and Families." Patient 10(2): 215-24.

LaVela, S. and A. Gallan. 2014. "Evaluation and Measurement of Patient Experience." Patient Experience Journal 1(1): 28-36.

Lees, C. 2011. "Measuring the Patient Experience." Nurse Research 19(1): 25-28.

Luxford, K., D.G. Safran and T. Delbanco. 2011. "Promoting Patient-Centered Care: A Qualitative Study of Facilitators and Barriers in Healthcare Organizations with a Reputation for Improving the Patient Experience." International Journal of Quality Health Care 23(5): 510-15.

Manary, M.P., W. Boulding, R. Staelin and S.W. Glickman. 2013. "The Patient Experience and Health Outcomes." New England Journal of Medicine 368(3): 201-03.

Marengoni, A., S. Angleman, R. Melis, F.Mangialasche, A. Karp, A. Garmen, et al. 2011. "Aging with Multimorbidity: A Systematic Review of the Literature." Ageing Research Review 10(4): 430-39. 
Nelson, M.L., A. Grudniewicz and S. Albadry. 2016. "Applying Clinical Practice Guidelines to the Complex Patient: Insights for Practice and Policy from Stroke Rehabilitation." Healthcare Quarterly 19(2): 38-43.

Osborn, R., D. Moulds, E.C. Schneider, M.M. Doty, D. Squires and D.O. Sarnak. 2015. "Primary Care Physicians in Ten Countries Report Challenges Caring for Patients with Complex Health Needs." Health Affairs 34(12): 2104-12.

Paddison, C.A., C.L. Saunders, G.A. Abel, R.A. Payne, J.L. Campbell and M. Roland. 2015. "Why Do Patients with Multimorbidity in England Report Worse Experiences in Primary Care? Evidence from the General Practice Patient Survey." BMJ Open 5(3): e006172.

Patton, M.Q., K. McKegg and N. Wehipeihana. 2015. Developmental Evaluation Exemplars: Principles in Practice. New York, NY: Guilford Publications.

Perkins, M., V.J. Howard, V.G. Wadley, M. Crowe, M.M. Safford, W.E. Haley, et al. 2013. "Caregiving Strain and All-Cause Mortality: Evidence from the REGARDS Study." Journals of Gerontology: Series B, Psychological Sciences and Social Sciences 68(4): 504-12.

Reinders, H. 2010. "The Importance of Tacit Knowledge in Practices of Care." Journal of Intellectual Disability Research 54(Suppl. 1): 28-37.

Robert, G. 2013. 'Participatory Action Research: Using Experience-Based Co-design to Improve the Quality of Healthcare Services." In Ziebland, S., A.C. Joseph, D. Calabrese, L. Locock, eds., Understanding and Using Health Experiences (pp. 138-49). Oxford, UK: Oxford University Press.

Roydhouse, J.K. and I.B. Wilson. 2017. "Systematic Review of Caregiver Responses for Patient HealthRelated Quality of Life in Adult Cancer Care." Quality of Life Research 26(8): 1925-54. doi:10.1007/ s11136-017-1540-6.
Salisbury, C. 2012. "Multimorbidity: Redesigning Health Care for People Who Use It." Lancet 380(9836): 7-9.

Sawatzky, R., S. Bryan, S. Cohen, A. Gadermann, K. Schick Makaroff and K. Stajduhar. 2015. Patient and Family Reported Experience and Outcome Measures for Use in Acute Care: A Knowledge Synthesis. Final Report. Kingston, ON: Canadian Frailty Network.

Sinha, S. 2013. Spotlight on Canadians: Results from the General Social Survey: Portrait of Caregivers, 2012. Ottawa, ON: Statistics Canada. Retrieved August 2, 2016. <http://www.statcan.gc.ca/pub/89-652-x/89652-x2013001-eng.htm>.

Smith, P., E. Mossialos. I. Papanicolas and S. Leatherman. 2008. Performance Measurement for Health System Improvement: Experiences, Challenges and Prospects. Cambridge, UK: Cambridge University Press.

Tinetti, M.E., T.R. Fried and C.M. Boyd. 2012. "Designing Health Care for the Most Common Chronic Condition - Multimorbidity." Journal of the American Medical Association 307(23): 2493-94.

Tipper, B. 2010. A Scan of Existing and Planned Surveys of Patient/Client or Caregiver Experiences in Transitions across Providers in Ontario. Toronto, ON: Change Foundation.

Turcotte, M. 2013. Family Caregiving: What Are the Consequences? Ottawa, ON: Statistics Canada. Retrieved June 21, 2017. <http://www.statcan.gc.ca/ pub/75-006-x/2013001/article/11858-eng.htm>.

Vogeli, C., A.E. Shields, T.A. Lee, T.B. Gibson, W.D. Marder, K.B. Weiss et al. 2007. "Multiple Chronic Conditions: Prevalence, Health Consequences, and Implications for Quality, Care Management, and Costs." Journal of General Internal Medicine 22(Suppl. 3): 391-95.

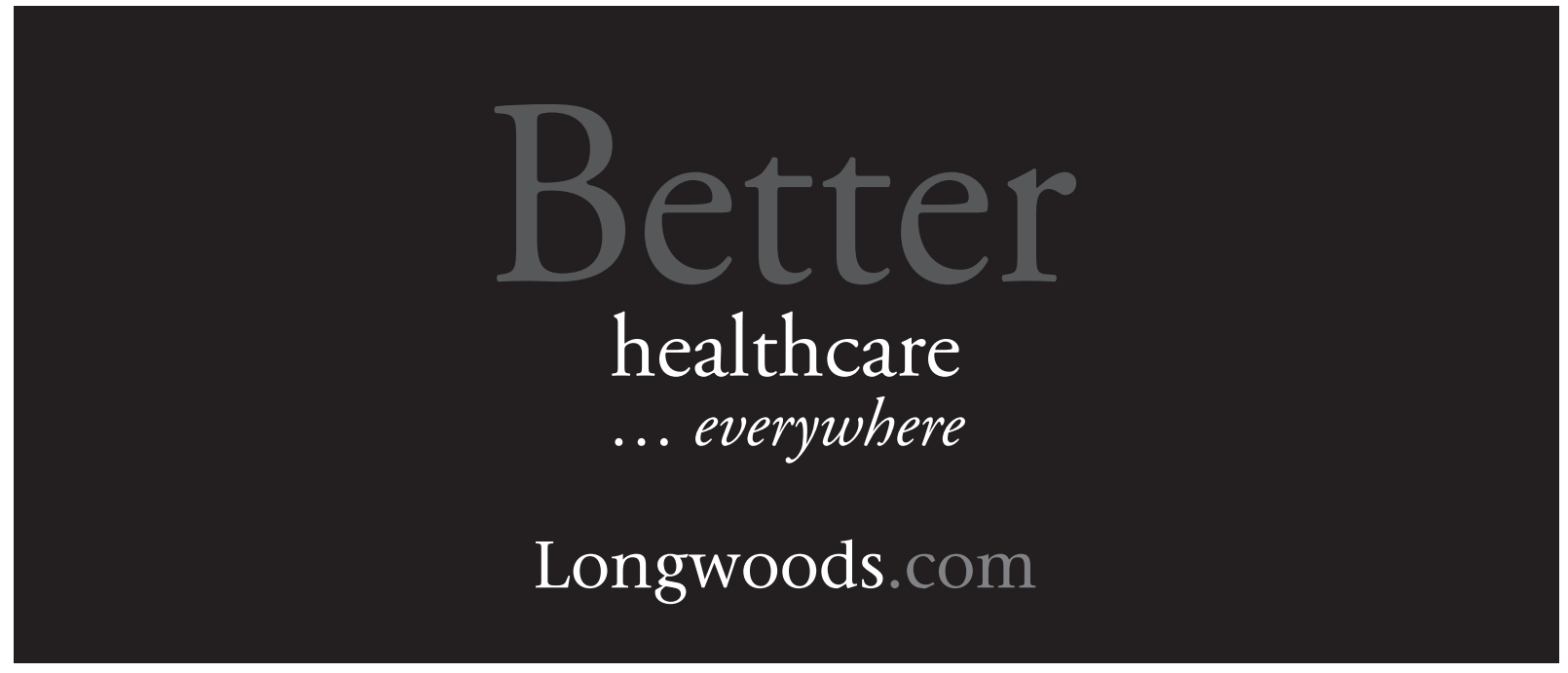

